\title{
Two-photon microscopy with simultaneous standard and extended depth of field using a tunable acoustic gradient-index lens
}

\author{
Nicolas Olivier, ${ }^{1}$ Alexandre Mermillod-Blondin, ${ }^{2}$ Craig B. Arnold, ${ }^{2}$ and Emmanuel Beaurepaire ${ }^{1, *}$ \\ ${ }^{1}$ Laboratory for Optics and Biosciences, Ecole Polytechnique, CNRS, INSERM, F-91128 Palaiseau, France \\ ${ }^{2}$ Department of Mechanical and Aerospace Engineering, Princeton University, Princeton, New Jersey 08544, USA \\ *Corresponding author: emmanuel.beaurepaire@polytechnique.edu
}

Received March 27, 2009; accepted April 24, 2009;

posted May 7, 2009 (Doc. ID 109299); published May 28, 2009

\begin{abstract}
We describe a simple setup that allows depth of field switching at kilohertz rates in a nonlinear microscope. Beam profile and/or divergence are modulated using a tunable, acoustically driven gradient-index fluid lens. We demonstrate two modulation strategies, one based on fast varifocus scanning during each pixel and the other based on pseudo-Bessel beam excitation. Average beam shape is switched every line during scanning, resulting in the interlaced acquisition of two different images. We apply this approach to the simultaneous standard and 4.5×-extended depth-of-field imaging of developing embryos. () 2009 Optical Society of America

OCIS codes: $180.4315,140.3300,230.1040,110.1080,180.6900,170.3880$.
\end{abstract}

Two-photon microscopy [1] is widely used for highresolution biological imaging, providing micrometer optical sectioning in complex samples. Optical slices are typically recorded by scanning the focused laser beam inside the sample. When needed, a more global representation of the sample can alternatively be obtained by using large depth-of-field optics, such as focused Bessel beams [2,3] produced by axicons [4,5], phase masks [6], or liquid-crystal-based spatial-light modulators [7]. However, these wavefront control strategies are either fixed or too slow to allow for rapid modulation between the two imaging regimes during a scan. Therefore, diffraction-limited and extended-depth images must be acquired sequentially.

We explore here an alternative approach. Using rapidly adjustable acousto-optic fluid lens technology, it is possible to construct a light-efficient imaging method with the ability of depth of field switching at kilohertz rates.

Imaging with shaped beams is performed as follows (see Fig. 1). The output beam from a Ti:sapphire oscillator is sent through a tunable, acoustically driven gradient-index (TAG) fluid lens [8-11], providing phase modulation. The TAG lens consists of a cylindrical piezoelectric shell driven at ultrasonic frequencies $(100-800 \mathrm{kHz})$ to generate standing pressure waves in a transparent filling fluid (silicone oil). In turn, the acoustic wave induces a periodic alteration of the refractive index inside the lens with fixed radial nodes [8,9]. Galvanometric mirrors angularly scan the beam at the pupil of a waterimmersion objective, and two-photon-excited fluorescence (2PEF) is epidetected using a photon-counting photomultiplier.

To acquire two simultaneous images, we use an interlaced acquisition scheme in which each line is scanned twice, and the modulation amplitude of the acoustic lens is switched between lines during galvanometer flyback. We typically acquire odd lines with near-zero modulation amplitude (off), and even lines with 10-21 V amplitude sine modulation (on). A stable pattern in the TAG is achieved in a few tens of microseconds, i.e., before the next line starts. The two images are deinterlaced after acquisition.

In a first configuration, we use the TAG lens as a varifocus device (Fig. 2). The TAG lens is positioned at the output of the Ti:sapphire laser where the beam is smallest, so that it experiences the refractive index distribution only near the center of the lens, which can be approximated in cylindrical coordinates by $[10]$

(a)

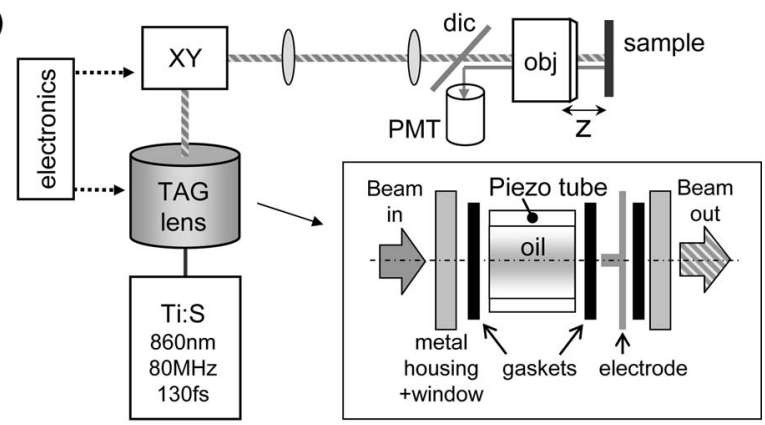

(b)

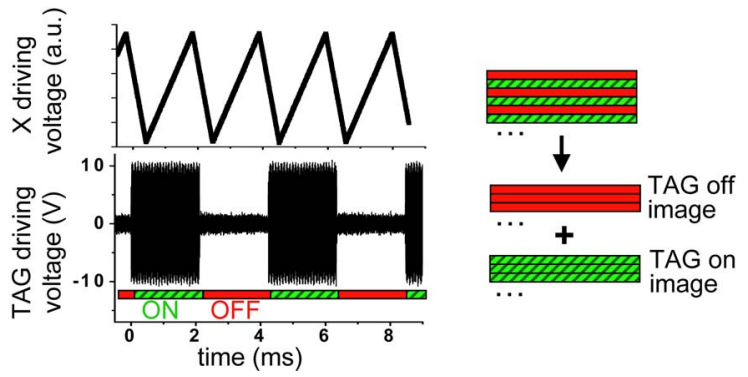

Fig. 1. (Color online) (a) Microscopy with acoustically modulated beams. TAG lens, tunable acoustic gradientindex lens (inset). XY, lateral scanning; dic, dichroic; PMT, photomultiplier; obj, $20 \times 0.95$ NA objective with underfilled pupil. (b) Principle of the interlaced acquisition with standard (off) and modulated (on) beams. 
(a)

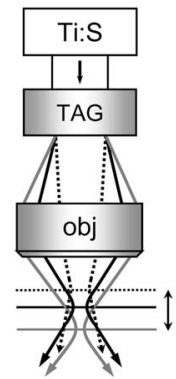

(d)

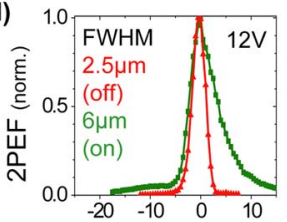

(b)

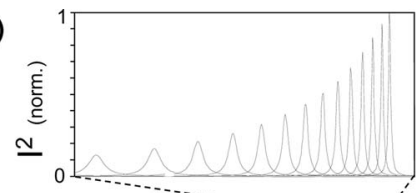

(c)
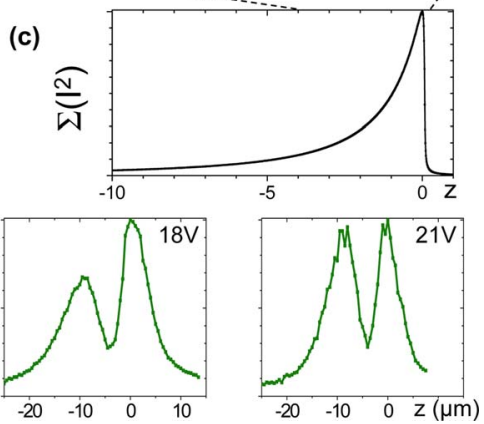

Fig. 2. (Color online) Point-spread functions (PSFs) obtained when using the TAG lens as a fast varifocus device. (a) Principle: beam divergence at the objective pupil is rapidly modulated during one pixel acquisition time. (b), (c) Numerical simulations of the instantaneous (b) and timeaveraged (c) on-axis intensity distribution. (d) Experimental axial 2PEF PSFs recorded from $330 \mathrm{~nm}$ beads switches from $2.5 \mu \mathrm{m}$ (lens off) to $6 \mu \mathrm{m}$ (on) FWHM and exhibits a two-lobe distribution when applying large modulation amplitudes (see text).

$$
n(\rho, t)=n_{0}+\left(n_{a} \frac{n_{a} \omega^{2} \rho^{2}}{4 v^{2}}\right) \sin (\omega t)
$$

where $\omega$ is the driving frequency, $n_{0}$ is the static index of refraction, $v$ is the speed of sound in the acoustic medium, and $n_{a}$ depends on the medium and the modulation parameters. The lens is located $\approx 1 \mathrm{~m}$ before the galvanometric mirrors and is driven at a frequency of $481 \mathrm{kHz}$. The pixel rate is set to $240 \mathrm{kHz}$, meaning that each pixel is the sum of $\approx 330$ pulses, effectively covering the entire focal range of the lens twice per pixel. Each of these pulses sees the TAG as a fixed lens with optical power ranging from $-\delta_{\max }$ to $+\delta_{\max }$, resulting in variable beam divergence at the objective pupil. This system can be simply modeled under Gaussian paraxial approximation. Two regimes must be considered. In the first case, the focal length of the acoustic lens is always larger than the distance to the objective. The effective point-spread function (PSF) is then the incoherent superposition of instantaneous PSFs with different axial positions, widths, and intensities, resulting in the broad asymmetric distribution qualitatively predicted in Fig. 2(c) and experimentally illustrated in the first panel of Fig. 2(d), where $\delta_{\max }<2 \mathrm{~m}^{-1}$. (Simulation parameters, $\delta_{\max }=1 \mathrm{~m}^{-1}$; beam waist $w=1 \mathrm{~mm}$; objective $f$ $=2 \mathrm{~mm}$.) A second regime occurs if the focal range of the acoustic lens is increased up to where it focuses the beam before the objective, and a second axial lobe builds up. This regime is reached by increasing the modulation amplitude, as illustrated in the remaining panels of Fig. 2(d), where $\delta_{\max }>4 \mathrm{~m}^{-1}$.

The resulting PSF is significantly extended, but it exhibits an axial gap. This issue can be avoided by using the acoustic lens as a pseudo-Bessel beam generator. This is achieved here by driving the TAG lens at a higher frequency $(740 \mathrm{kHz})$ and by expanding the beam twice so that it fills a larger part of the lens and propagates through a refractive index distribution that can be described as [11]

$$
n(\rho, t)=n_{0}+n_{a} J_{0}\left(\frac{\omega \rho}{v}\right) \sin (\omega t) .
$$

As in the previous experiment, the laser and the piezo are not synchronized, meaning that successive pulses experience different modulations. The resulting time-averaged intensity is a multiscale Bessel beam with a transverse profile exhibiting a central peak surrounded by successive rings [11], which may be viewed as intermediate between a Gaussian and a Bessel beam. The position of the TAG lens relative to the microscope is adjusted so that the propagated unshaped beam [off, Fig. 3(a)] and the first major ring [on, Fig. 3(b)] have similar sizes at the objective pupil. When the TAG is on, all the instantaneous PSFs are axially elongated, and their incoherent superposition resembles that of a focused Bessel beam. Figure 3 displays images of $330 \mathrm{~nm}$ fluorescent beads in a 3D gel recorded with and without an extended depth of field. Beam shaping results in a 4.5-fold axial increase of the PSF without degrading lateral resolution ( $\sim 0.6 \mu \mathrm{m}$ FWHM, not shown), characteristic of Bessel-like excitation. In turn, extended depth of field results in more visible beads.

Finally, we show that this technique can be used for two-photon imaging of biological samples in a
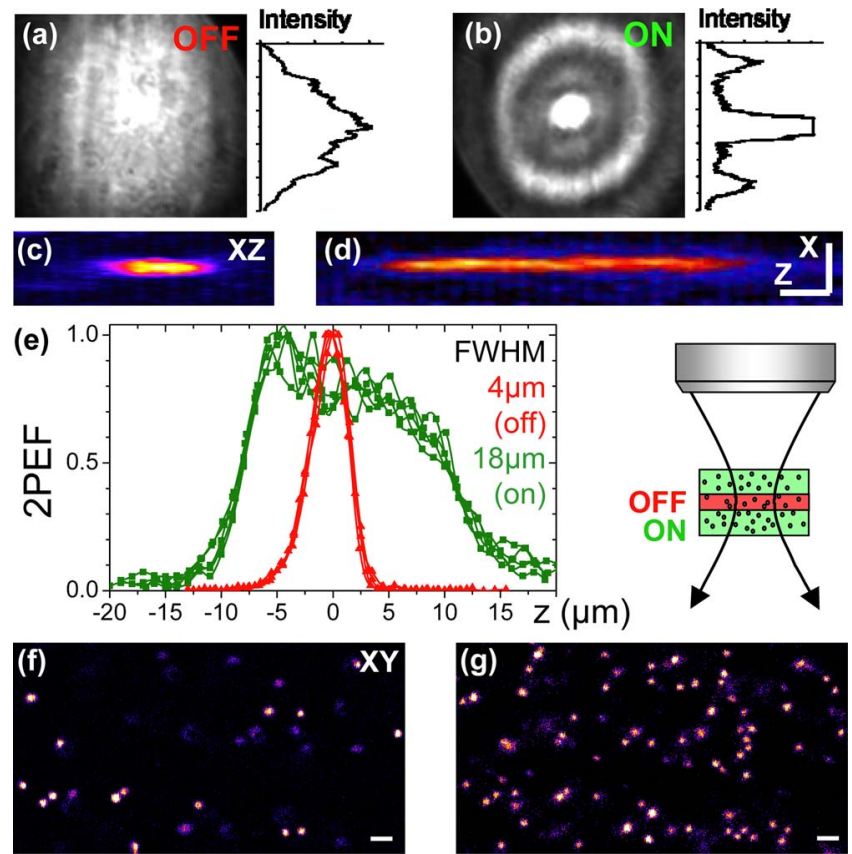

Fig. 3. (Color online) TAG lens used as a pseudo-Bessel beam generator. (a), (b) Average intensity distribution at the back aperture of the objective with the lens off and on. (c), (d) $Z-X, 2 P E F$ images of $330 \mathrm{~nm}$ fluorescent beads recorded with standard [(c), off)] and extended [(d), on] depth of field. (e) $z$ profiles through several bead images. FWHM are $4.0 \pm 0.2 \mu \mathrm{m}$ (off) and 18.3 $\pm 0.4 \mu \mathrm{m}$ (on). (f), (g) Beads in a 3D gel imaged with standard and extended depth of field. Scale bars, $3 \mu \mathrm{m}$. 
straightforward manner. Figures 4(a1) and 4(a2) present simultaneous standard and extended-depth images of a pollen grain recorded in two different planes separated by $12 \mu \mathrm{m}$. Although the optically sectioned images are very different, the corresponding extended-depth images obtained simultaneously with pseudo-Bessel excitation are similar and provide a more global view of the sample. Figures 4(b1) and 4b2) (Media 1) illustrate the fast switching capability of the TAG lens, which makes it suitable for imaging dynamic samples. Here, a developing Drosophila embryo with green-fluorescent-protein-labeled nuclei is observed continuously in a single plane during gastrulation. We oriented the embryo with the ventral side up (facing the objective) so as to image
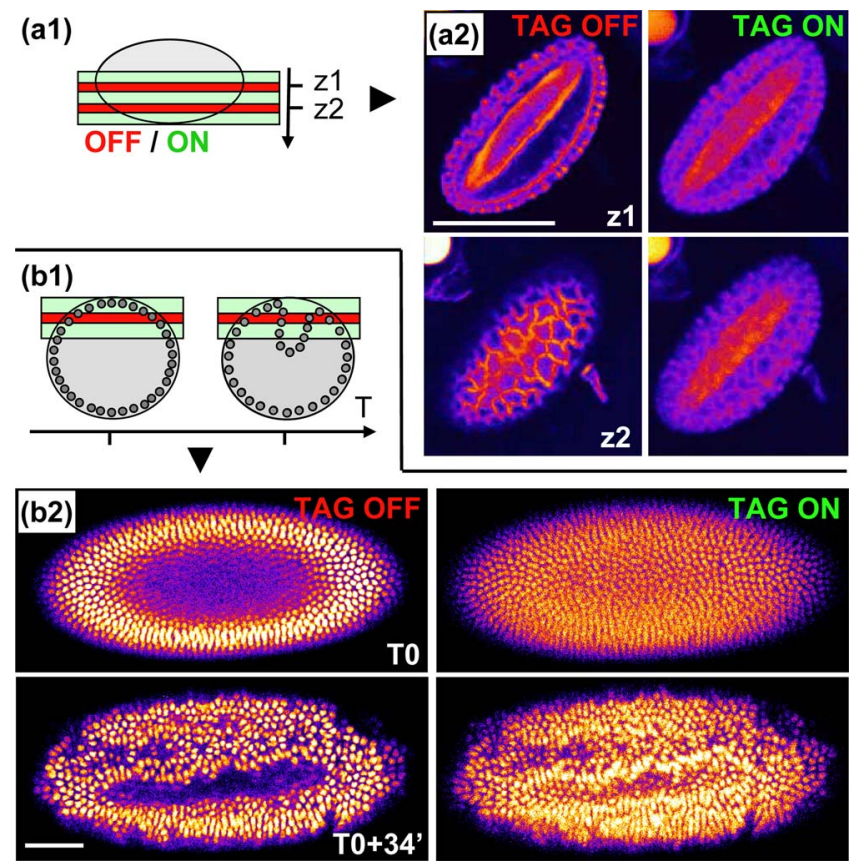

Fig. 4. (Color online) Two-photon imaging of a developing Drosophila embryo and of a pollen grain with simultaneous standard and extended depth of field. (a1), (a2) Images of a fixed pollen grain recorded at axial locations separated by $12 \mu \mathrm{m}$. The standard (off) images provide optical slices, whereas the simultaneously recorded extended-field images (on) provide a more global view of the sample. (b1), (b2) (Media 1) Time-lapse imaging of a developing Drosophila embryo with GFP-labeled cell nuclei, during ventral furrow formation. The beam focus was kept just below the outer cells of the ventral side, as illustrated in (b1). The standard movie shows the invaginating ventral cells passing through the focal plane, whereas the simultaneous extended-depth movie provides a global view of lateral cell movements during furrow formation. See Media 1. Scale bars, $50 \mu \mathrm{m}$. the formation of the ventral furrow, an early process involving cell invagination toward the middle of the embryo. The imaging plane is positioned just below the outer cells, so that early invaginating cells show up progressively in the standard time-lapse image. The extended-depth image provides a complementary global representation of the tissue that could not be obtained simultanesouly using slow or static beammodulation devices.

In conclusion, we have shown than an acoustically driven fluid lens can provide kilohertz switching rates between standard and extended-depth imaging in two-photon microscopy. This arrangement is simple, inexpensive, and light-efficient (90\% transmission over the $750-880 \mathrm{~nm}$ range). Furthermore, acoustic lenses can produce more-complex beam shapes, such as multiscale Bessel beams [11], and can be synchronized with the pulse train for increased wavefront control when using kilohertz laser systems. Additional perspectives include imaging with tunable depth-of-field and spatial control of multiphoton processes for imaging and sampleprocessing applications.

We thank X. Solinas for assistance in electric interfacing, E. McLeod for many discussions, and D. Débarre for comments on the manuscript. This work was partially supported by the Délégation Générale pour l'Armement (DGA), the Agence Nationale de la Recherche (ANR), and the U.S. Air Force Office of Scientific Research (AFOSR).

\section{References}

1. W. Denk, J. H. Strickler, and W. W. Webb, Science 248, 73 (1990).

2. J. Durnin, J. J. Miceli, and J. H. Eberly, Phys. Rev. Lett. 58, 1499 (1987).

3. D. McGloin and K. Dholakia, Contemp. Phys. 46, 15 (2005).

4. P. Dufour, M. Piché, Y. De Koninck, and N. McCarthy, Appl. Opt. 45, 9246 (2006).

5. Z. Ding, H. Ren, Y. Zhao, J. S. Nelson, and Z. Chen, Opt. Lett. 27, 243 (2002).

6. E. J. Botcherby, R. Juskaitis, and T. Wilson, Opt. Commun. 268, 253 (2006).

7. J. A. Davis, C. S. Tuvey, O. Lopez-Coronado, J. Campos, M. J. Yzuel, and C. Iemmi, Opt. Lett. 32, 844 (2007).

8. K. A. Higginson, M. A. Costolo, and E. A. Rietman, Appl. Phys. Lett. 84, 843 (2004).

9. E. McLeod and C. B. Arnold, J. Appl. Phys. 102, 033104 (2007).

10. A. Mermillod-Blondin, E. McLeod, and C. B. Arnold, Opt. Lett. 33, 2146 (2008).

11. E. McLeod and C. B. Arnold, Appl. Opt. 47, 3609 (2008). 